\title{
Release of Ciprofloxacin and Moxifloxacin From Daily Disposable Contact Lenses From an In Vitro Eye Model
}

\author{
Magdalena Bajgrowicz, ${ }^{1}$ Chau-Minh Phan, ${ }^{2}$ Lakshman N. Subbaraman, ${ }^{2}$ and Lyndon Jones ${ }^{2}$ \\ ${ }^{1}$ Department of Mechanics, Material Science and Engineering, Wroclaw University of Technology, Wroclaw, Poland \\ ${ }^{2}$ Centre for Contact Lens Research, School of Optometry and Vision Science, Waterloo, Ontario, Canada
}

Correspondence: Chau-Minh Phan, Centre for Contact Lens Research, School of Optometry and Vision Science, 200 University Avenue West, Waterloo, ON N2L 3G1, Canada; c2phan@uwaterloo.ca.

Submitted: January 5, 2015

Accepted: February 27, 2015

Citation: Bajgrowicz M, Phan C-M, Subbaraman LN, Jones L. Release of ciprofloxacin and moxifloxacin from daily disposable contact lenses from an in vitro eye model. Invest $O p h$ thalmol Vis Sci. 2015;56:2234-2242. DOI:10.1167/iovs.15-16379
Purpose. To analyze the release of two fluoroquinolones, ciprofloxacin and moxifloxacin, from conventional hydrogel $(\mathrm{CH})$ and silicone hydrogel $(\mathrm{SH})$ daily disposable contact lenses (CLs), comparing release from a fixed-volume vial and a novel in vitro eye model.

Methods. Four CH CLs (nelfilcon A, omafilcon A, etafilcon A, ocufilcon B) and three SH CLs (somofilcon A, narafilcon A, delefilcon A) were used. The lenses were incubated in drug solutions for 24 hours. After the incubation period, the lenses were placed in two release conditions: (1) a vial containing $4.8 \mathrm{~mL}$ PBS for 24 hours and (2) an in vitro eye model with a flow rate at $4.8 \mathrm{~mL}$ over 24 hours.

Results. Release in the vial for both drugs was rapid, reaching a plateau between 15 minutes and 2 hours for all lenses. In contrast, under physiological flow conditions, a constant and slow release was observed over 24 hours. The amounts of ciprofloxacin released from the lenses ranged between $49.6 \pm 0.7$ and $62.8 \pm 0.3 \mu \mathrm{g}$ per lens in the vial, and between $35.0 \pm$ 7.0 and $109.0 \pm 5.0 \mu \mathrm{g}$ per lens in the eye model. Moxifloxacin release ranged from $24.0 \pm$ 4.0 to $226.0 \pm 2.0 \mu \mathrm{g}$ per lens for the vial, and between $13.0 \pm 2.0$ and $151.0 \pm 10.0 \mu \mathrm{g}$ per lens in the eye model. In both systems and for both drugs, HEMA-based CLs released more drugs than other materials.

Conclusions. The parameters of the release system, in particular the volume and flow rate, have a significant influence on measured release profiles. Under physiological flow, release profiles are significantly slower and constant when compared with release in a vial.

Keywords: ciprofloxacin, moxifloxacin contact lens, drug delivery, eye model, microfluidic, silicone hydrogel

\begin{abstract}
Ciprofloxacin and moxifloxacin are important antimicrobial $\checkmark$ agents for the treatment of a wide range of ocular surface diseases. ${ }^{1-4}$ Both drugs are examples of fluoroquinolone agents, with a wide range of activity against both gram-negative and gram-positive bacteria., 2,3 Their popularity in treating ocular infection is attributed to their broad spectrum of inhibition, excellent ocular penetration, and their availability in a wide form of ophthalmic preparations. ${ }^{2,3}$ Fluoroquinolones prevent bacterial DNA multiplication by inhibiting two enzymes that are essential for chromosome replication: DNA gyrase and DNA topoisomerase IV.
\end{abstract}

Ciprofloxacin is the most widely prescribed second-generation quinolone antibiotic, which came into clinical use in the late 1980s. ${ }^{1}$ This relatively hydrophobic drug has low solubility at physiological $\mathrm{pH}(0.09 \mathrm{mg} / \mathrm{mL}$ at $\mathrm{pH} 7)$ and increased solubility in acidic or basic mediums $(3.46 \mathrm{mg} / \mathrm{mL}$ at $\mathrm{pH} 5) .{ }^{1}$ It is currently available in the form of $0.3 \%$ eye drops or $0.3 \%$ ointments. Moxifloxacin is a newer, fourth-generation 8-quinolone ${ }^{5}$ with an extended treatment spectrum, as well as increased bacteriostatic and bactericidal efficacy, when compared with ciprofloxacin. ${ }^{6-9}$ The drug has superior penetration into the anterior ocular tissues, and has greater resistance to bacterial efflux mechanisms, due to its bulky side chain moiety. ${ }^{9}$ Overall, this allows the drug to accumulate more effectively inside bacterial cells. Furthermore, simultaneous mutation to both enzymes by the pathogen of interest are required to decrease the efficacy of moxifloxacin, while only one mutation is sufficient to increase the resistance of pathogens against ciprofloxacin. ${ }^{10}$ Finally, another notable improvement of moxifloxacin compared with its predecessor is improved water solubility at $\mathrm{pH} 7(24 \mathrm{mg} /$ $\mathrm{mL}) .{ }^{11}$ It is currently prescribed as $0.5 \%$ eye drops for ophthalmic applications.

Eye drops, although overall convenient with regard to formulation preparations, are very inefficient as a treatment regimen, as average drug residence time on the ocular surface is only 2 to 5 minutes. ${ }^{12}$ The estimated amount of medicine that is absorbed by the cornea during that time is only $1 \%$ to $7 \%$ of the applied dose, and the rest is effectively washed out by continuous tear flow from the lacrimal sac into the nasolacrimal duct, where it is absorbed into the bloodstream. ${ }^{13}$ This may lead to undesirable systemic side effects. Moreover, to achieve therapeutic drug concentrations in the target tissues, drugs must be applied very frequently in small doses, even during sleeping hours. ${ }^{14,15}$ This type of treatment regimen results in both discomfort and inconvenience for the patient. Therefore, there is a considerable need for a drug delivery device that can effectively deliver drugs to the ocular surface for an extended period of time.

The use of contact lenses (CLs) may be a promising approach to deliver drugs to the cornea. ${ }^{16,17}$ Since 1965 , CLs have been proposed as potential drug carriers, ${ }^{17,18}$ and a great deal of research has been conducted since then to develop a 
TABLE 1. Properties of CH Lenses Used in the Study

\begin{tabular}{lllll}
\hline & Biomedics 1-Day & 1-Day Acuvue Moist & Proclear 1 Day & DAILIES AquaComfort Plus \\
\hline US adopted name & Ocufilcon B & Etafilcon A & Omafilcon A & Nelfilcon A \\
Manufacturer & CooperVision & Johnson \& Johnson & CooperVision & Alcon \\
Water content, \% & 52 & 58 & 60 & 69 \\
FDA group & IV & IV & II & II \\
Center thickness, mm & 0.07 & 0.08 & 33.0 & 0.10 \\
Oxygen permeability, $\times 10^{-11}$ & 16.8 & 28.0 & HEMA, PC, EGDMA & 26.0 \\
Principal monomers & HEMA, PVP, MA & HEMA, MA & PVA, FMA, PEG
\end{tabular}

EGDMA, ethylene glycol dimethacrylate; FMA, N-formylmethyl acrylamide; PVP, polyvinyl pyrrolidone.

commercial product. ${ }^{19-21}$ Unfortunately, studies in the past have shown that unmodified, commercially available CLs containing drugs rapidly release their drug load within the first hour. ${ }^{22-24}$ For this reason, commercial CLs were considered poor candidates for prolonged drug delivery. However, previous in vitro models used to measure release rates may not have reflected the physical parameters found in the ocular environment. For instance, the tear volume on the corneal surface is approximately $7 \pm 2 \mu \mathrm{L},{ }^{25}$ which undergoes a rate of tear exchange at approximately 0.95 to $1.55 \mu \mathrm{L} / \mathrm{min}^{26}$ To date, much of the published research has performed release experiments by immersing the lenses in vials containing 2 to $5 \mathrm{~mL}$ PBS, with no form of fluid exchange. ${ }^{22,23,27}$ Because the mechanism of drug release from unmodified CLs can be described as primarily simple diffusion, it should not be surprising that drugs are eluted very quickly when exposed to large volumes of static fluid. The in vivo release of drugs from CLs on the ocular surface, which has a lower volume and constant tear turnover, may be much slower.

To provide a better understanding of the drug release mechanisms in vivo, we developed an in vitro eye model that simulates both tear volume and flow. This study highlights the differences between the standard large volume, static vial model previously used by researchers, and our in vitro eye model, by evaluating the release kinetics of ciprofloxacin (relatively hydrophobic) and moxifloxacin (relatively hydrophilic) from four commercially available daily disposable conventional $(\mathrm{CH})$ hydrogel lenses and three silicone hydrogel $(\mathrm{SH})$ lens materials.

\section{Materials and Methods}

\section{Contact Lenses}

Seven commercially available disposable CLs were used in this study: four CH CLs (nelfilcon A [Alcon, Fort Worth, TX, USA], omafilcon A [CooperVision, Scottsville, NY, USA], etafilcon A [Johnson \& Johnson, Jacksonville, FL, USA], and ocufilcon B [CooperVision]) and three SH lenses (somofilcon A [CooperVision], narafilcon A [Johnson \& Johnson], and delefilcon A
[Alcon]). The lenses were obtained in their original packaging, and each lens had a dioptric power of -3.00 and base curve of 8.5 or $8.6 \mathrm{~mm}$. The properties of the lenses are listed in Tables 1 and 2.

\section{Preparation of Drug Solutions and Incubation}

Six lenses of each type were incubated for 24 hours in $3.0 \mathrm{mg}$ / $\mathrm{mL}$ ciprofloxacin solution (Sigma-Aldrich, Oakville, Ontario, Canada) in PBS. The $\mathrm{pH}$ for this drug solution was adjusted using hydrochloric acid to $\mathrm{pH} 4.0$ to solubilize the drug. Another six lenses of each type were incubated in $1.0 \mathrm{mg} / \mathrm{mL}$ of moxifloxacin (Selleckchem, Houston, TX, USA) solution in PBS (pH 7.4) for 24 hours.

\section{Eye Model Release}

For the purpose of this study, a novel eye model was created. Solid works 2013 was used to design the eye model as a computer-aided drawing file that was printed using threedimensional (3-D) printing technology. The resulting 3-D printed polycarbonate-acrylonitrile-butadiene-styrene molds, consisting of a "corneal/scleral" section and an "eye lid" piece, can be filled with the desired polymer. In this study, these molds were filled with polydimethylsiloxane and cured at $75^{\circ} \mathrm{C}$ for 1 hour to obtain the eye model. The spacing between the eyeball and eyelid allows for approximately $100 \mu \mathrm{L}$ fluid to come in contact with the lens. The eye models are connected to a microfluidic syringe pump (PHD Ultra; Harvard Apparatus, Holliston, MA, USA), as shown in Figure 1.

After the incubation period, lenses were partially dried on lens paper and placed into the eye model. Then PBS was pumped through the model at a rate of $200 \mu \mathrm{L} / \mathrm{h}$. The flowthrough fluid was collected in a standard 12-well microliter plate. At specified time intervals, $t=0,1,2,3,4,5,6,8,12,16$, and 24 hours, $200 \mu \mathrm{L}$ of this solution was withdrawn and pipetted into a UV- Star transparent plate (Greiner Bio-One, Frickenhausen, Germany) and measured using the SpectraMax M5 UV-Vis Spectrophotometer (Molecular Devices, Sunnyvale, CA, USA). The concentration of ciprofloxacin was measured

TABLE 2. Properties of SH Lenses Used in the Study

\begin{tabular}{llll}
\hline & DAILIES TOTAL1 & & 1-DAY ACUVUE TruEye \\
\hline US adopted name & Delefilcon A & Narafilcon A & Somofilcon A \\
Manufacturer & Alcon & Johnson \& Johnson & CooperVision \\
Water content, \% & $33($ surface $>80 \%)$ & 46 & 56 \\
FDA group & $\mathrm{V}$ & $\mathrm{V}$ & $\mathrm{V}$ \\
Center thickness, $\mathrm{mm}$ & 0.09 & 0.09 & 0.07 \\
Oxygen permeability, $\times 10^{-11}$ & 140 & 100 & 60 \\
Oxygen transmissibility, $\times 10^{-9}$ & 156.0 & 118.0 & 86.0 \\
Principal monomers & Not disclosed & MPMDSM, DMA, HEMA, siloxane macromer, TEGDMA, PVP & Not disclosed
\end{tabular}

DMA, N,N-dimethylacrylamide; MPDMS, monofunctional polydimethylsiloxane; TEGDMA, tetraethyleneglycol dimethacrylate. 
A

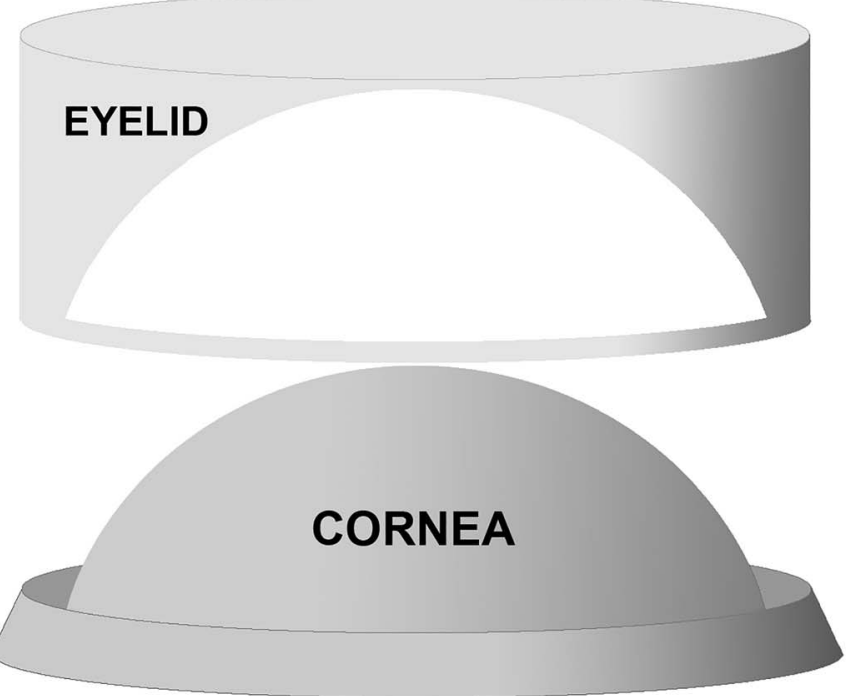

C

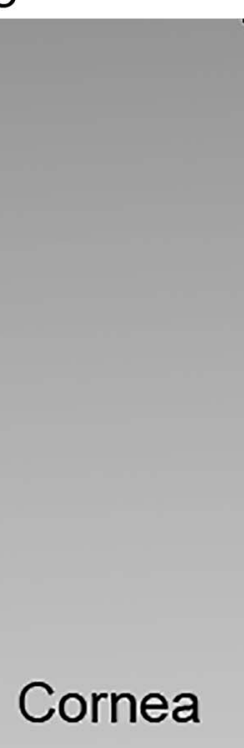

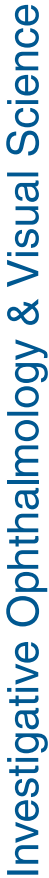

\section{Eyelid}

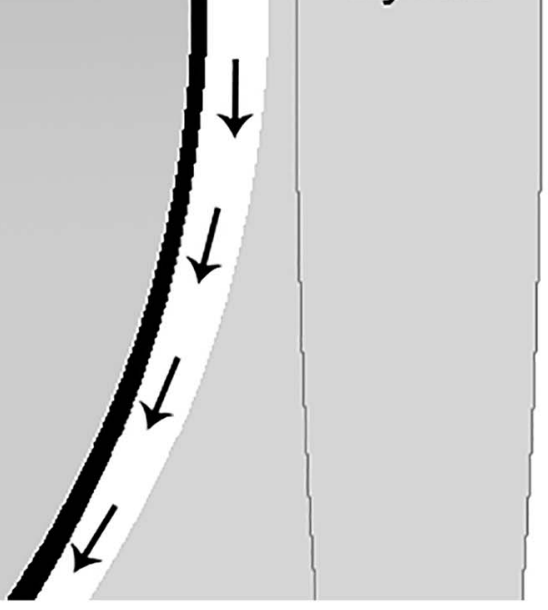

B

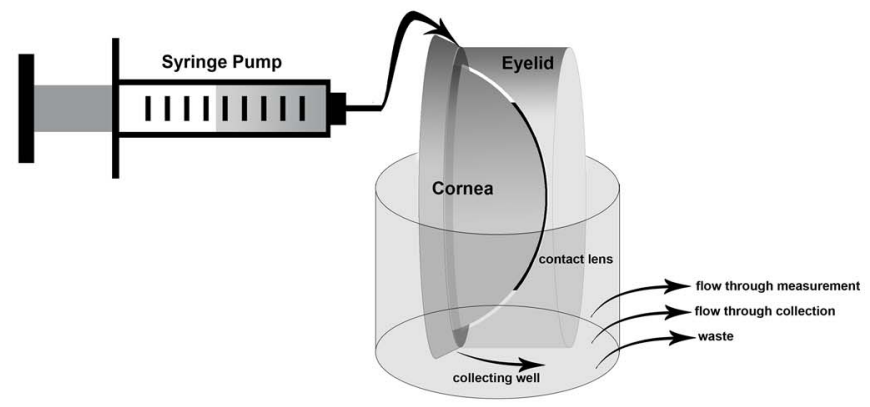

D

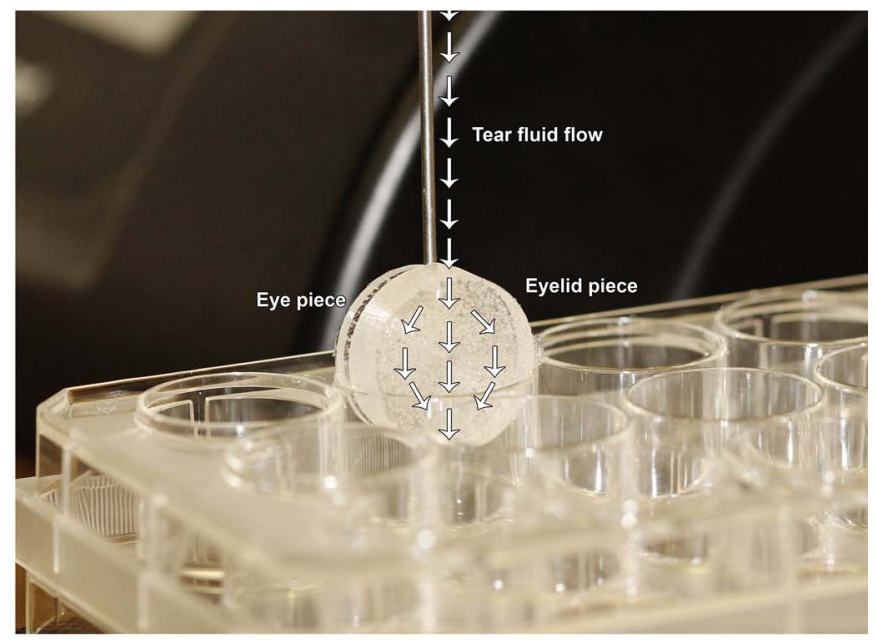

Figure 1. Schematic of (A) two-piece eye model. (B) Final set up with a microfluidic pump and a collecting well plate and (C) a close-up showing how the tear fluid flows over the lens on the eye model. (D) A front-view photograph of the eye model. 
TABLE 3. Release ( $\mu \mathrm{g} / \mathrm{lens})$ of Ciprofloxacin and Moxifloxacin after 24 Hours From CH and SH Daily Disposable CLs

\begin{tabular}{|c|c|c|c|c|c|}
\hline Commercial Name & Material & $\begin{array}{c}\text { Ciprofloxacin } \\
\text { in Vial, } \mu \mathrm{g} / \mathrm{Lens}\end{array}$ & $\begin{array}{c}\text { Ciprofloxacin } \\
\text { in Eye Model, } \mu \mathrm{g} / \text { Lens }\end{array}$ & $\begin{array}{c}\text { Moxifloxacin } \\
\text { in Vial, } \mu \mathrm{g} / \text { Lens }\end{array}$ & $\begin{array}{c}\text { Moxifloxacin } \\
\text { in Eye Model, } \mu \mathrm{g} / \text { Lens }\end{array}$ \\
\hline${ }^{\mathrm{CH}}$ 1-Day Acuvue Moist & Etafilcon A & $62.4 \pm 0.6$ & $109 \pm 5$ & $226 \pm 2$ & $151 \pm 10$ \\
\hline${ }^{\mathrm{CH}}$ Biomedics 1-Day & Ocufilcon B & $62.8 \pm 0.3$ & $106 \pm 2$ & $218 \pm 4$ & $150 \pm 20$ \\
\hline${ }^{\mathrm{CH}}$ Proclear 1 Day & Omafilcon A & $60.9 \pm 0.3$ & $96 \pm 5$ & $141 \pm 2$ & $86 \pm 8$ \\
\hline${ }^{\mathrm{CH}}$ DAILIES AquaComfort Plus & Nelfilcon A & $55.2 \pm 0.4$ & $35 \pm 7$ & $37 \pm 4$ & $35 \pm 6$ \\
\hline${ }^{\mathrm{SH}}$ clariti 1 day & Somofilcon A & $56.9 \pm 0.2$ & $63 \pm 6$ & $36 \pm 1$ & $35 \pm 3$ \\
\hline${ }^{\mathrm{SH}}$ DAILIES TOTAL1 & Delefilcon A & $49.6 \pm 0.7$ & $49 \pm 1$ & $24 \pm 4$ & $20 \pm 1$ \\
\hline${ }^{\mathrm{SH}}$ 1-DAY ACUVUE TruEye & Narafilcon A & $52.9 \pm 0.9$ & $40 \pm 5$ & $33 \pm 3$ & $13 \pm 2$ \\
\hline
\end{tabular}

using fluorescence, with excitation and emission wavelengths at 274 and $451 \mathrm{~nm}$, respectively. The concentration of moxifloxacin was measured using absorbance at $287 \mathrm{~nm}^{28} \mathrm{~A}$ standard curve for the drug concentration versus fluorescence was generated in PBS for ciprofloxacin. For moxifloxacin, a standard curve for drug concentration versus absorbance was generated in PBS. The samples were diluted as necessary such that the fluorescence and absorbance values were within the linear portion of the standard curve.

\section{Vial Release}

After the 24-hour drug incubation, lenses were removed from the drug solution and partially dried on lens paper. The lenses were then placed in a vial containing $4.8 \mathrm{~mL}$ solution of PBS, at $\mathrm{pH}$ 7.4. At specific time intervals, $t=0,1,5,15$, and 30 minutes, and $1,2,4,8,12,16$, and 24 hours, $200 \mu \mathrm{L}$ of the sample was withdrawn from the vial and pipetted into a UVStar transparent plate (Greiner Bio-One). After each absorbance or fluorescence was measured using the SpectraMax M5 UV-Vis Spectrophotometer (Molecular Devices), the sample solutions were pipetted back into their respective vials.

\section{Statistical Analysis}

Statistical analysis was performed using Statistica 8 software (StatSoft, Tulsa, OK, USA). All data are reported as mean \pm SD, unless otherwise stated. Repeated measures ANOVA was performed to determine the differences across various time points within the same lens material. An ANOVA was conducted to determine the differences between lens materials at each time point. Post hoc Tukey multiple comparison tests were used when necessary. In all cases, values were considered statistically significant at $P$ less than 0.05 . Graphs were plotted using GraphPad Prism 5 software (GraphPad, La Jolla, CA, USA).

\section{Results}

The total amount of ciprofloxacin and moxifloxacin released after 24 hours from seven daily disposable CLs are summarized in Table 3. For both drugs, there were differences in the total amount of drug released after 24 hours from the vial compared with the model eye $(P<0.001)$. The release profile over 24 hours from the vial and in vitro eye model are illustrated in Figures 2 to 5. In general, hydroxyethyl methacrylate (HEMA)based $\mathrm{CH}$ lenses released more ciprofloxacin and moxifloxacin compared with SH lenses $(P<0.001)$, when measured using either the vial or the model eye.

For ciprofloxacin, there was a difference in the total amount of drugs released after 24 hours from the vial compared with the eye model $(P<0.001)$. Nelfilcon A had a lower drug release in the model eye $(P<0.05)$, whereas omafilcon A, etafilcon A, and ocufilcon $\mathrm{B}$ had a higher drug release from the model eye $(P<0.001)$. There was no significant difference in the release of ciprofloxacin from the $\mathrm{SH}$ lenses between the two release systems. In the vial, most of the drug release occurred within the first 2 hours and plateaued thereafter $(P<0.05)$, as shown in Figure 3 . Narafilcon A (TruEye; Johnson \& Johnson) was the only lens

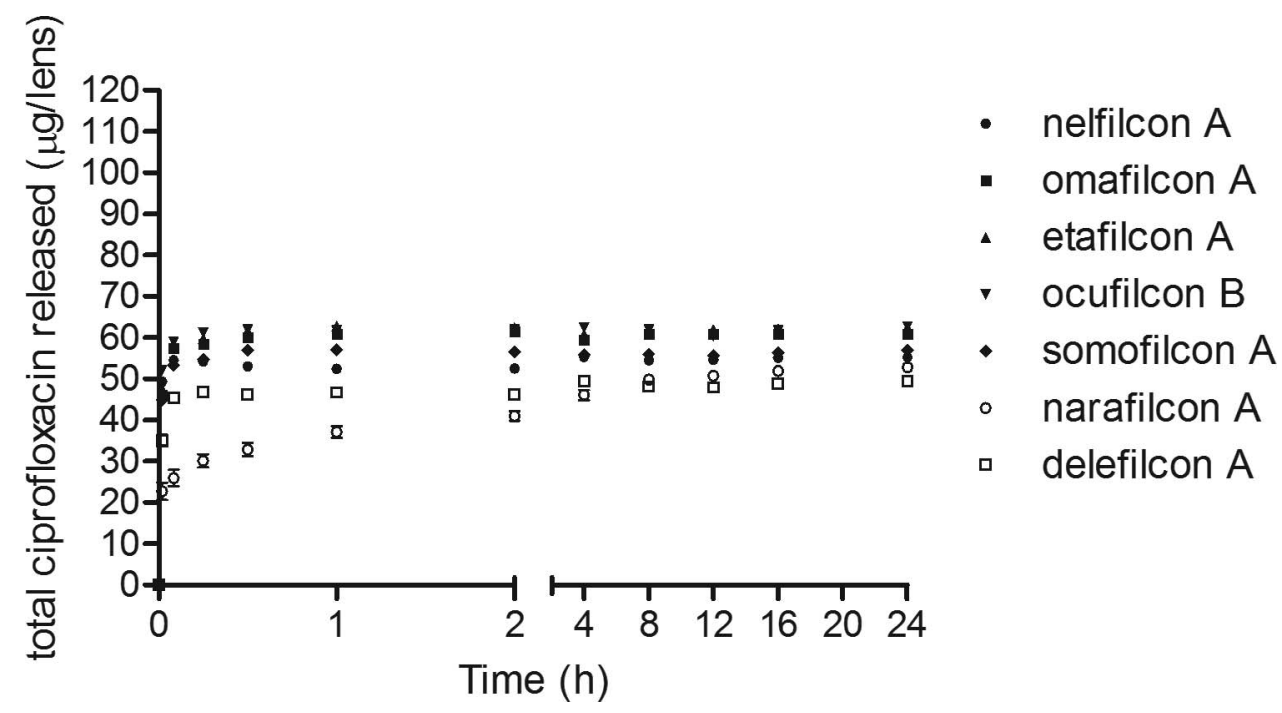

Figure 2. Release of ciprofloxacin ( $\mu \mathrm{g} / \mathrm{lens})$ from daily disposable commercial CLs in $4.8 \mathrm{~mL}$ PBS. The values plotted are the mean \pm SD for three trials. 


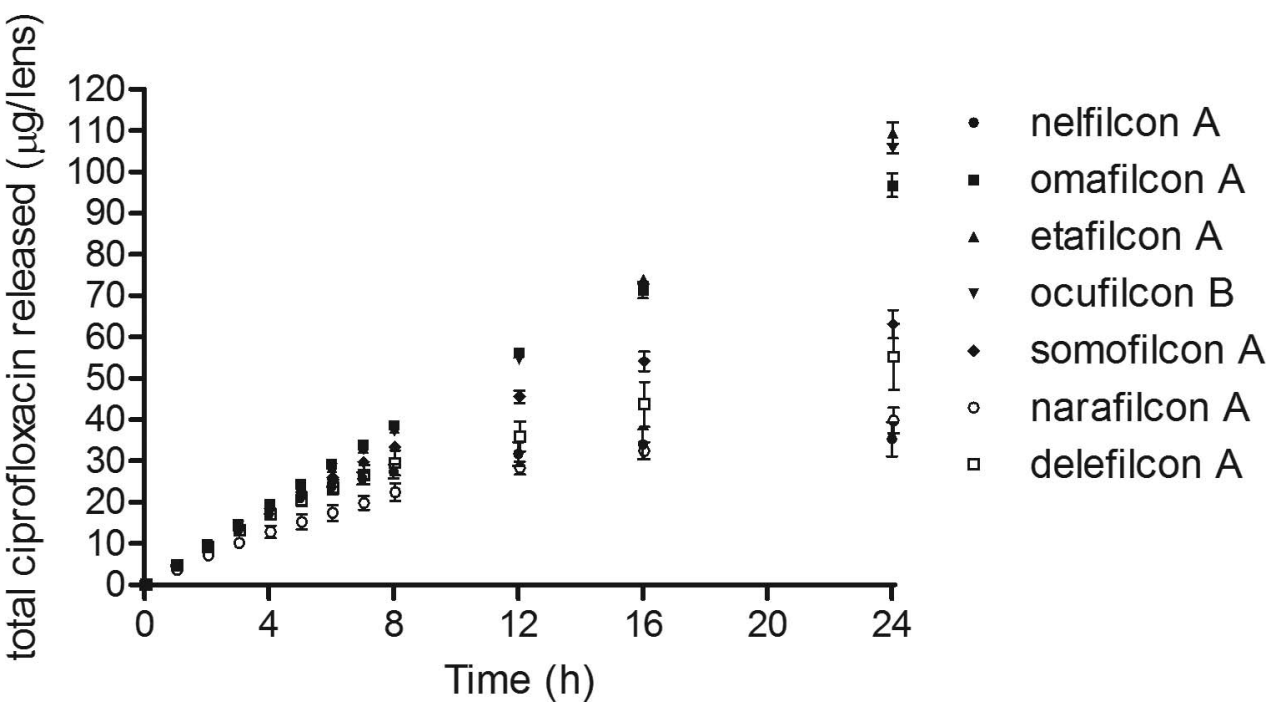

Figure 3. Release of ciprofloxacin ( $\mu \mathrm{g} / \mathrm{lens})$ from eye model with a flow rate of $4.8 \mathrm{~mL}$ over 24 hours. The values plotted are the mean \pm SD for three trials.

in which an 8-hour drug release was observed in this system. In contrast, as shown in Figure 4, the drug release was sustained from the eye model throughout the 24-hour observation period $(P<0.05)$. The lenses that released the highest amount of ciprofloxacin in both systems were omafilcon A, etafilcon A, and ocufilcon B. However, as shown in Figure 6, these lenses had a high amount of white drug precipitates and thus may not be suitable as ocular drug delivery lenses. The SH CLs with ciprofloxacin remained clear throughout the experiment.

For moxifloxacin, after 24 hours, the release from the vial was overall higher than the eye model $(P<0.001)$. Omafilcon $\mathrm{A}$, etafilcon $\mathrm{A}$, and ocufilcon $\mathrm{B}$ had a higher drug release in the vial than the eye model $(P<0.001)$. There were no significant differences in the amount of moxifloxacin released for the other lenses between the two systems. In the vial model, the release of the moxifloxacin rapidly reached a plateau within 1 hour for all SH lenses and nelfilcon A $(P<0.001)$. Etafilcon A and ocufilcon $\mathrm{B}$ continued to release the drug up to 2 hours before reaching a plateau. In contrast, in the eye model, the release of moxifloxacin was sustained over 24 hours for etafilcon A and ocufilcon B $(P<0.001)$. Omafilcon A released at a constant rate of approximately $10 \mu \mathrm{g} / \mathrm{h}$ for 6 hours, after which the release slowed down to $2 \mu \mathrm{g} / \mathrm{h}$. Nelfilcon A and SH lenses reached a plateau in less than 6 hours $(P<0.05)$. The other lenses released moxifloxacin for less than 4 hours, after which time the rate plateaued.

In both experimental systems, materials that released the highest amounts of moxifloxacin were etafilcon $\mathrm{A}$ and ocufilcon B (which are both HEMA-based, Food and Drug Administration [FDA] group IV materials with a high water content and are negatively charged), followed by omafilcon A (a HEMA-based, FDA group II material with a high water content but overall neutral charge). Materials that released the lowest amount of drug in both systems were nelfilcon A (a polyvinyl alcohol [PVA]-based, FDA group II material with a high water content and neutral charge), and all $\mathrm{SH}$ lenses. Visually, all of the lenses containing moxifloxacin remained clear throughout all phases of the experiment.

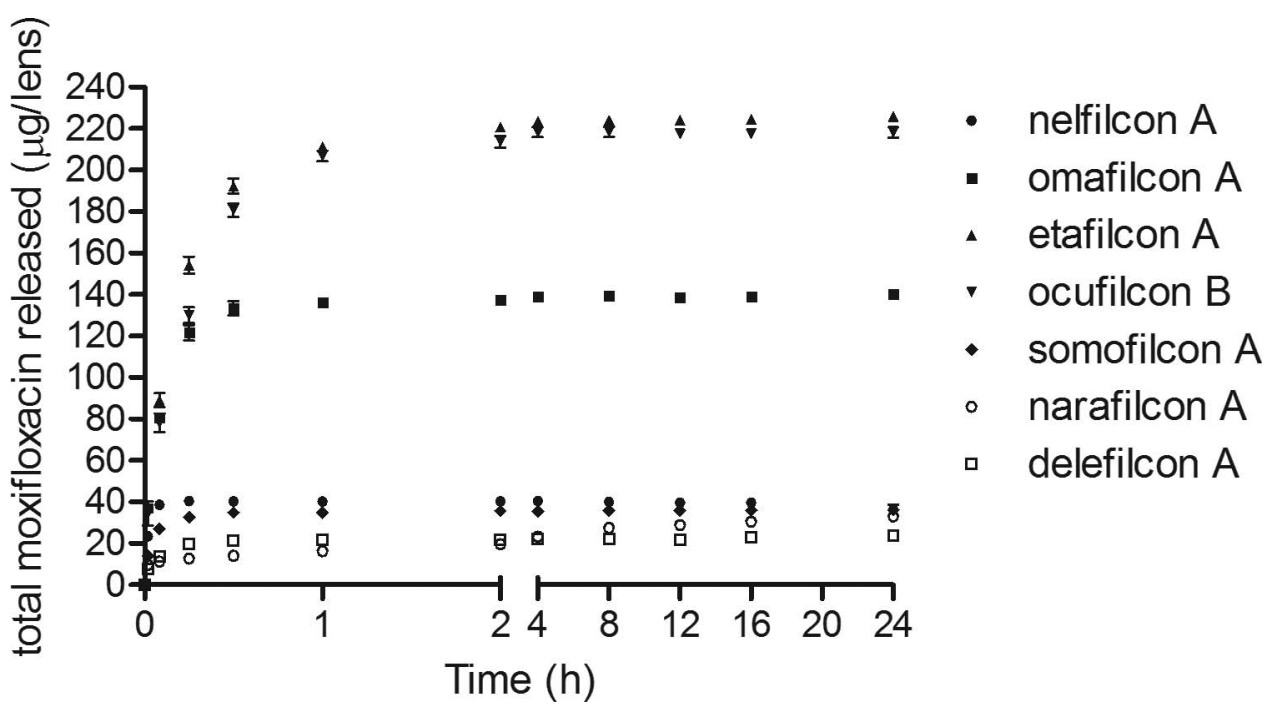

Figure 4. Release of moxifloxacin ( $\mu \mathrm{g} / \mathrm{lens}$ ) from daily disposable commercial CLs in $4.8 \mathrm{~mL}$ PBS. The values plotted are the mean \pm SD for three trials. 


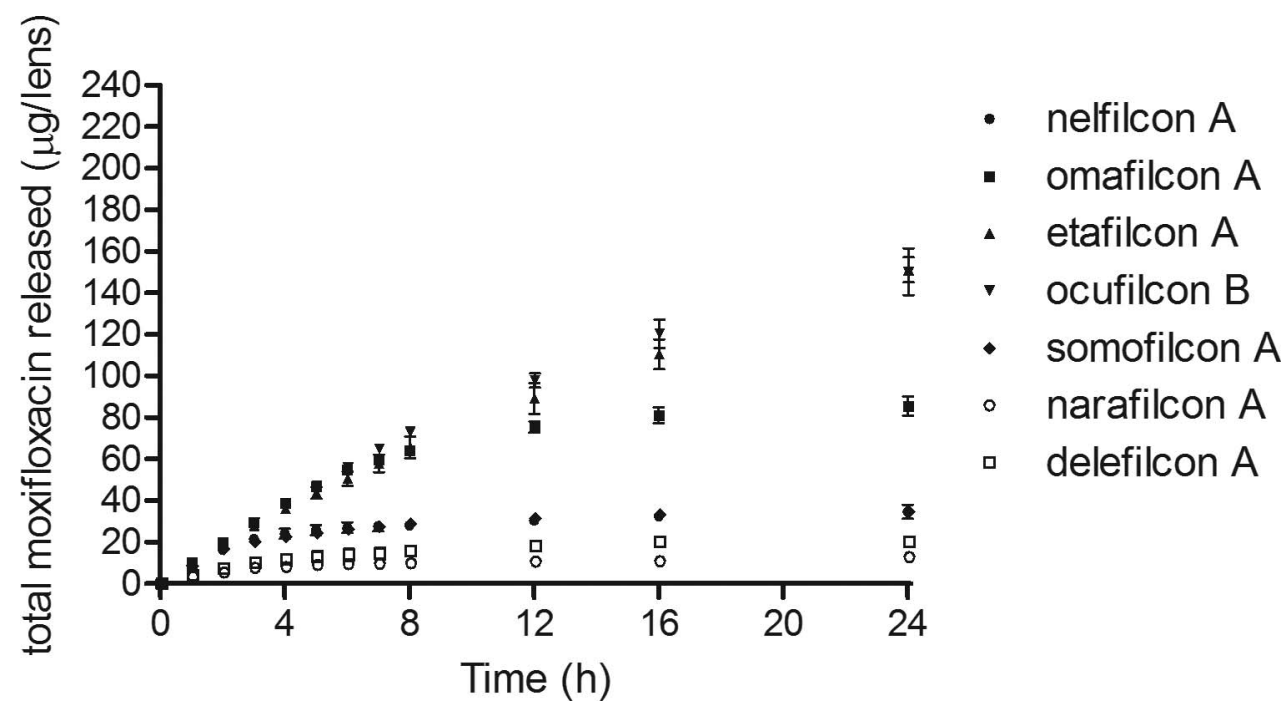

Figure 5. Release of moxifloxacin ( $\mu \mathrm{g} / \mathrm{lens})$ from eye model with a flow rate of $4.8 \mathrm{~mL}$ over 24 hours. The values plotted are the mean \pm SD for three trials.

\section{Discussion}

The ideal CL device for prolonged drug delivery should be able to deliver a precise amount of drug over an extended period of time. Standard release measurements with unmodified com- mercial lenses showed that commercial CLs would generally release drugs too rapidly to be clinically useful. ${ }^{22-24}$ Similar results were observed in our study, as CLs loaded with either ciprofloxacin or moxifloxacin would release their contents within 1 hour when immersed in a vial containing $4.8 \mathrm{~mL}$

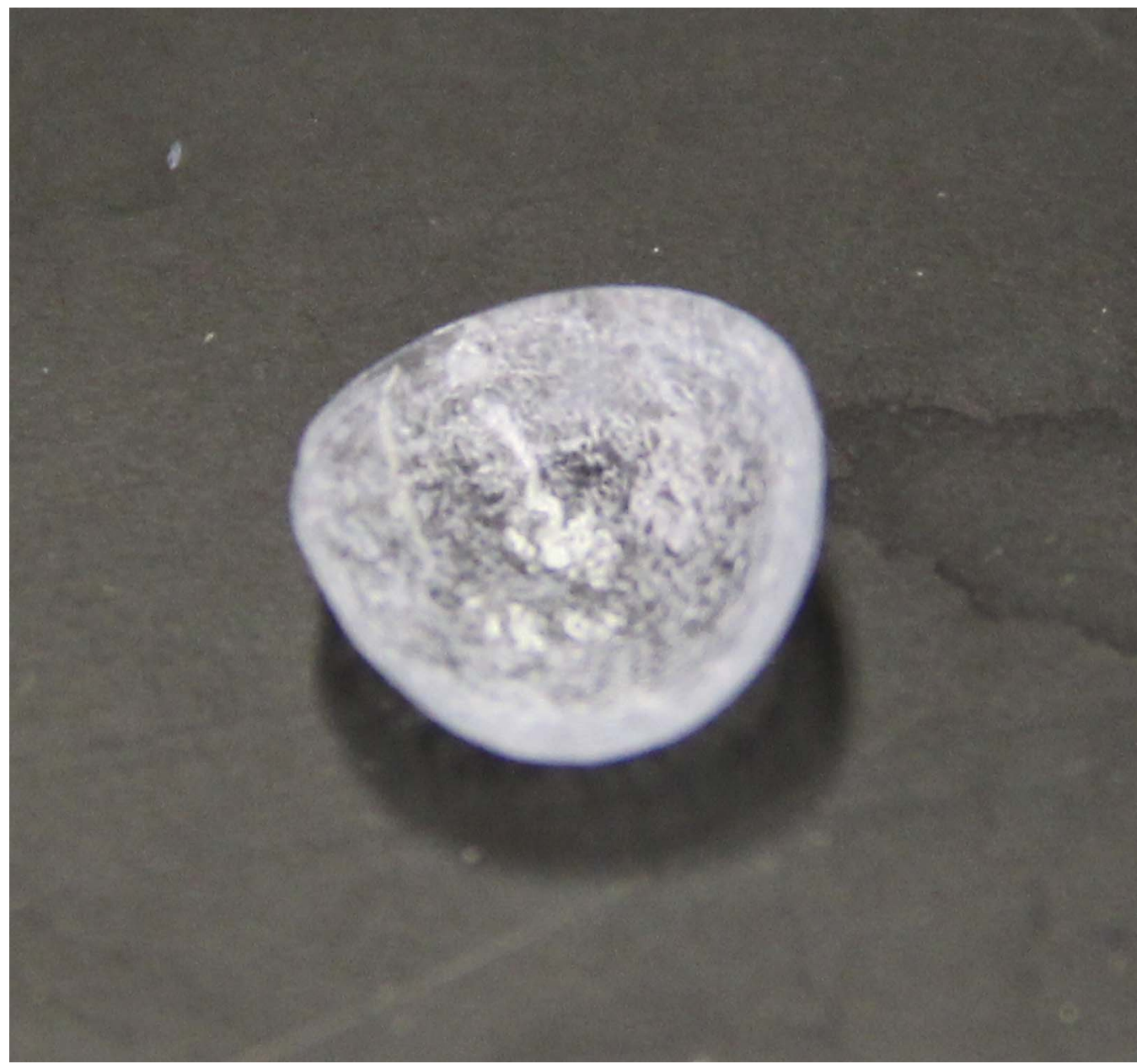

Figure 6. Ciprofloxacin precipitates on $\mathrm{CH}$ lenses (omafilcon A, etafilcon A, ocufilcon B). 
saline. However, closer examination of this release setup clearly reveals that the parameters used are far from similar to those found on the ocular surface. In particular, there is an absence of natural tear flow (at $0.95-1.55 \mu \mathrm{L} / \mathrm{min}$ ), ${ }^{26}$ and the volume in a vial far exceeds that of the ocular surface, which has a tear film volume of $7 \pm 2 \mu \mathrm{L} .{ }^{25}$ Because the release is controlled primarily by diffusion of the drug from the CL, these parameters are crucial in correctly simulating in vivo release kinetics.

The in vitro eye model presented in this study is a two-piece model, consisting of a corneal/scleral section and an eyelid component. These two components, when pushed together, allow for the simulation of a thin tear film layer, approximately $100 \mu \mathrm{L}$ in volume. The added microfluidic system to this model allows for the simulation of physiological tear flow conditions. As seen from the results, under physiological conditions, there was no sign of burst drug release, and the overall release was also significantly slower. Similar results have been observed from other studies that have examined drug release from CLs in microfluidic systems. ${ }^{29-32}$ The model presented in this study represents a closed eye and lacks a blinking mechanism. It has recently been shown that the effect of eye blinking can facilitate drug release from HEMA hydrogels, and thus should be considered as an important factor affecting ocular drug delivery. ${ }^{33}$

In addition to the differences in release profiles between the two systems, there were also differences in the amounts of drugs released during 24 hours. Notably, for moxifloxacin, lenses released up to $40 \%$ more drug in a vial than in the eyesimulation model. This difference may be accounted for by the fact that the release from the lenses in the eye model may not have yet reached completion, even after 24 hours. The experiment was performed for only 24 hours because all of the CLs used in this experiment were daily disposable CLs, and thus should not be worn for more than 24 hours. However, the results suggest that the drugs could probably be eluted from the lenses for a longer period of time. In contrast, the release of ciprofloxacin from HEMA-based $\mathrm{CH}$ lenses in a vial was surprisingly lower than in the eye model. The release of the drug plateaued entirely in the vial-based system after 2 hours, but visible white precipitation on the lens surfaces indicates that a substantial quantity of drug still remained within the material. Although the mechanism is uncertain, the release likely ceased as the drug concentration in the CL and the release media reached an equilibrium. In the eye model, equilibrium was not reached due to the continual tear flow, and as a result a higher amount of drug was eluted.

Overall, the CLs that released the highest amount of drugs were HEMA-based $\mathrm{CH}$ lenses. Etafilcon A and ocufilcon $\mathrm{B}$ had the highest drug release, followed by omafilcon A. Etafilcon A and ocufilcon B consist of HEMA, PVA, and methacrylic acid (MA), whereas omafilcon A is made from HEMA and PC (2methacryloyloxyethyl phosphorylcholine). The results suggest that materials synthesized from copolymers of MA, such as ocufilcon B and etafilcon A, tend to release higher amounts of drug than materials without MA The addition of MA results in a negative charge for the lens material, which attracts positively charged amino groups of ciprofloxacin and moxifloxacin. This finding correlates with previous studies with ciprofloxacin, which also showed that the presence of MA in the polymer increases the amount of drugs absorbed and released. ${ }^{13}$

The results from this study indicate that $\mathrm{CH}$ lenses in general release more drugs than $\mathrm{SH}$ lenses, which is similar to results found in previous studies. ${ }^{22,24}$ The silicone moieties within SH lenses produce hydrophobic regions within the lens, which promotes the deposition of hydrophobic drugs, such as ciprofloxacin. However, the release of ciprofloxacin from these lenses into an aqueous environment is minimized because of the favorable hydrophobic-hydrophobic interaction between the lens and the drug. ${ }^{24}$ In addition, it also would be difficult for water to penetrate these hydrophobic pockets to elute the drugs. For hydrophilic drugs, such as moxifloxacin, sorption into $\mathrm{SH}$ lenses is unfavorable because of the overall polymer hydrophobicity. Consequently, because of the lesser amounts of drugs initially absorbed, SH lenses released less moxifloxacin overall than $\mathrm{CH}$ lenses. Furthermore, the weak interaction between the drug and the polymer resulted in faster elution of moxifloxacin from $\mathrm{SH}$ lenses. One particular material, nelfilcon A, was an exception to the observed trend. Although the material is inherently a conventional hydrogel, it showed a significantly lower release for both ciprofloxacin and moxifloxacin compared with other $\mathrm{CH}$ lenses. This mechanism is unclear, but could be linked to the unique wetting agents within the material. Wetting agents, such as PVA and polyethylene glycol (PEG), are not cross-linked to the polymer. Consequently, they occupy space within the polymer matrix, and reduce the ability of the lens to absorb certain substances, such as drugs, potentially leading to the lower drug release observed for nelfilcon A.

In this study, the difference in release kinetics between a relatively hydrophobic drug (ciprofloxacin) and relatively hydrophilic drug (moxifloxacin) also was examined. Using the large-volume vial method, there were no significant differences in release between the drugs, with both being released rapidly from the lens within the first hour. This observation is similar to results previously published by other researchers using the vial as the release system. ${ }^{22-24}$ However, using the in vitro eye model, we observed considerable differences in release profiles for these drugs. The elution of drugs into an aqueous media is determined by its water solubility, and the rate at which the drug reaches equilibrium between the lens and the solution. For ciprofloxacin, the solubility is low, ${ }^{1}$ and the constant flow rate prevents equilibrium from being reached. As a result, ciprofloxacin was released at a slow and sustained rate for all lens types over 24 hours. In contrast, moxifloxacin, which has much higher solubility, ${ }^{11}$ showed a much faster drug release. Etafilcon A and ocufilcon B were the only two lenses able to release moxifloxacin for 24 hours, whereas omafilcon A released all the drug within 12 hours. All SHs and narafilcon A were able to release moxifloxacin for only 4 hours in the eye model.

Both drugs showed the best release profile with CH HEMAbased lenses. Unfortunately, for ciprofloxacin, white drug precipitates formed on the surface of these lenses during the release phase, rendering them opaque. For this reason, despite showing ideal release kinetics, CH HEMA-based lenses are not compatible for ciprofloxacin delivery. The SH lenses, although releasing a lower quantity of drug, may be more suitable for ciprofloxacin because they provided sustained release for 24 hours, while remaining transparent.

Minimum inhibitory concentrations for $90 \%$ of bacterial isolates $\left(\mathrm{MIC}_{90}\right)$ is defined as the concentration required to inhibit the growth of $90 \%$ of organisms. The $\mathrm{MIC}_{90}$ for ciprofloxacin against common, susceptible, and resistant ocular pathogens ranges from 0.032 to $128 \mu \mathrm{g} / \mathrm{mL} .{ }^{34}$ All of the lenses released sufficient amount of drug to meet the $\mathrm{MIC}_{90}$ concentrations against common and susceptible pathogens during the entire 24-hour period. However, none of the lenses released enough drug to kill fluoroquinolone-resistant pathogens. For moxifloxacin, $\mathrm{MIC}_{90}$ ranges from 0.047 to $4 \mu \mathrm{g} / \mathrm{mL} .^{34}$ As in the case of ciprofloxacin, all of the lenses released enough drug to meet the $\mathrm{MIC}_{90}$ concentrations against common and susceptible pathogens over the 24-hour period. For fluoroquinolone-resistant pathogens, such as coagulase- 
negative Staphylococcus or Staphylococcus aureus, only etafilcon A, ocufilcon B, and omafilcon A were able to release enough drug to meet the $\mathrm{MIC}_{90}$ criteria for 24 hours. The remaining lenses were able to release sufficient drug for only 2 to 4 hours.

In conclusion, seven different daily disposable CLs were evaluated for their release of two fluoroquinolones, ciprofloxacin and moxifloxacin, in two different release systems. The results suggest that the release profiles are influenced primarily by the parameters of the release system. In a vial, drugs are released rapidly within the first hour, which is consistent with previous reports. However, using an in vitro eye model that mimics physiological tear volume and tear flow, drug release from CLs is observed at a much more constant rate over 24 hours. The release kinetics of drugs from CLs also is affected by material properties of the lens, as well as the hydrophobicity and hydrophilicity of the drug. Among all the drug-material combinations, the most promising was the release of moxifloxacin from etafilcon $\mathrm{A}$ and ocufilcon $\mathrm{B}$. The release was sustained throughout 24 hours, and the amount of eluted drug can be considered clinically relevant, as it meets the MIC $_{90}$ criteria against common, susceptible, and resistant ocular pathogens during this time period. Additionally, the lenses remained visually clear throughout all phases of the experiment. Future work will improve the in vitro eye model, and further examine the potential of these CLs to inhibit microbial growth.

\section{Acknowledgments}

Supported by NSERC 20/20 Network for the Development of Advanced Ophthalmic Materials (CMP, LNS, LJ) and Projekt Nowa jakość kształcenia na Wydziale Mechanicznym Politechniki Wrocławskiej współfinansowany ze środków Unii Europejskiej w ramach Europejskiego Funduszu Społecznego (MB)

Disclosure: M. Bajgrowicz, None; C.-M. Phan, None; L.N. Subbaraman, None; L. Jones, None

\section{References}

1. Ross D, Riley C. Aqueous solubilities of some variously substituted quinolone antimicrobials. Int J Pharm. 1990;63: 237-250.

2. Bower K, Kowalski R, Gordon Y. Fluoroquinolones in the treatment of bacterial keratitis. Am J Ophthalmol. 1996;121: 712-715.

3. Smith A, Pennefather P, Kaye S. Fluoroquinolones: place in ocular therapy. Drugs. 2001;61:747-761.

4. Marchese A, Slana V, Holmes E, Jay W. Toxicity and pharmacokinetics of ciprofloxacin. J Ocul Pharmacol. 1993; 9:69-76.

5. Stroman D, Dajcs J, Cupp G, Schlech B. In vitro and in vivo potency of moxifloxacin and moxifloxacin ophthalmic solution $0.5 \%$, a new topical fluoroquinolone. Surv Ophthalmol. 2005;50:16-31.

6. Johnson A, Warner M, Livermore D. Activity of moxifloxacin and other quinolones against pneumococci resistant to firstline agents, or with high-level ciprofloxacin resistance. Int J Antimicrob Agents. 2001;17:377-381.

7. Ince D, Zhang X, Hooper D. Activity of and resistance to moxifloxacin in Staphylococcus aureus. Antimicrob Agents Chemother. 2003;47:1410-1415.

8. Kowlski R, Dhaliwal D, Karenchak L, et al. Gatifloxacin and moxifloxacin: an in vitro susceptibility comparison to levofloxacin, ciprofloxacin, and ofloxacin using bacterial keratitis isolates. Am J Ophthalmol. 2003;136:500-505.
9. Pestova E, Millichap J, Noskin G, Peterson L. Intracellular targets of moxifloxacin: a comparison with other fluoroquinolones. J Antimicrob Chemother. 2000;45:583-590.

10. Spence R, Towner K. Frequencies and mechanisms of resistance to moxifloxacin in nosocomial isolates of Acinetobacter baumannii. J Antimicrob Chemother. 2003;52:687690.

11. Varanda F, Pratas de Melo M, Caco A, et al. Solubility of antibiotics in different solvents. 1. hydrochloride forms of tetracycline, moxifloxacin, and ciprofloxacin. Ind Eng Chem Res. 2006;45:6368-6374.

12. Gulsen D, Chauhan A. Ophthalmic drug delivery through contact lenses. Invest Ophthalmol Vis Sci. 2004;45:23422347.

13. Tangri T, Khurana S. Basics of ocular drug delivery systems. International Journal of Research in Pharmaceutical and Biomedical Sciences. 2011;2:1541-1548.

14. Lesher G, Gunderson G. Continuous drug delivery through the use of disposable contact lenses. Optom Vis Sci. 1993;70: 1012-1018.

15. Chrai S, Makoid M, Eriksen S, Robinson J. Drop size and initial dosing frequency problems of topically applied ophthalmic drugs. J Pharm Sci. 1974;63:333-338.

16. Hehl E, Beck R, Luthard K, Guthoff R, Drewelow B. Improved penetration of aminoglycosides and fluoroquinolones into the aqueous humour of patients by means of Acuvue contact lenses. Eur J Clin Pharmacol. 1999;55:317-325.

17. Sedlacek J. Possibilities of application of eye drugs with the aid of gel-contact lenses. Cesk Oftalmol. 1965;21:509-512.

18. Jain M. Drug delivery through soft contact lenses. $\mathrm{Br} J$ Ophthalmol. 1988;72:150-154.

19. Xinming L, Yingde C, Lloyd A, et al. Polymeric hydrogels for novel contact lens-based ophthalmic drug delivery systems: a review. Cont Lens Anterior Eye. 2008;31:57-64.

20. Ambroziak A, Szaflik J, Szaflik J. Therapeutic use of a silicone hydrogel contact lens in selected clinical cases. Eye Contact Lens. 2004;30:63-67.

21. Hyatt A, Rajan M, Burling K, Ellington M, Tassoni A, Martin K. Release of vancomycin and gentamicin from a contact lens versus a fibrin coating applied to a contact lens. Invest Ophthalmol Vis Sci. 2012;53:1946-1952.

22. Hui A, Boone A, Jones L. Uptake and release of ciprofloxacin$\mathrm{HCl}$ from conventional and silicone hydrogel contact lens materials. Eye Contact Lens. 2008;34:266-271.

23. Peng C, Kim J, Chauhan A. Extended delivery of hydrophilic drugs from silicone-hydrogel contact lenses containing vitamin E diffusion barriers. Biomaterials. 2010;31:4032-4047.

24. Phan CM, Subbaraman LN, Jones L. In vitro uptake and release of natamycin from conventional and silicone hydrogel contact lens materials. Eye Contact Lens. 2013;39:162-168.

25. Mishima S, Gasset A, Klyce S, Baum J. Determination of tear volume and tear flow. Invest Ophthalmol Vis Sci. 1966;5:264276.

26. Furukawa R, Polse K. Changes in tear flow accompanying aging. Am J Optom Physiol Opt. 1978;55:69-74.

27. Boone A, Hui A, Jones L. Uptake and release of dexamethasone phosphate from silicone hydrogel and group I, II, and IV hydrogel contact lenses. Eye Contact Lens. 2009;35:260-267.

28. Motwani S, Chopra S, Ahmad F, Khar R. Validated spectrophotometric methods for the estimation of moxifloxacin in bulk and pharmaceutical formulations. Spectrochim Acta A Mol Biomol Spectrosc. 2007;68:250-256.

29. Tieppo A, Pate K, Byrne M. In vitro controlled release of an anti-inflammatory from daily disposable therapeutic contact lenses under physiological ocular tear flow. Eur J Pharm Biopharm. 2012;81:170-177. 
30. Ali M, Horikawa S, Venkatesh S, Saha J, Hong J, Byrne M. Zeroorder therapeutic release from imprinted hydrogel contact lenses within in vitro physiological ocular tear flow. J Control Release. 2007;124:154-162.

31. White C, McBride M, Pate K, Tieppo A, Byrne M. Extended release of high molecular weight hydroxypropyl methylcellulose from molecularly imprinted, extended wear silicone hydrogel contact lenses. Biomaterials. 2011;32:5698-5705.

32. Kaczmarek J, Tieppo A, White C, Byrne M. Adjusting biomaterial composition to achieve controlled multiple-day release of dexamethasone from an extended-wear silicone hydrogel contact lens. J Biomater Sci Polym Ed. 2014;25:88100.

33. Galante R, Paradiso P, Moutinho MG, et al. About the effect of eye blinking on drug release from pHEMA-based hydrogels: an in vitro study. J Biomater Sci Polym Ed. 2015;26:235251.

34. Oliveira A, D'Azevedo P, Francisco W. In vitro activity of fluoroquinolones against ocular bacterial isolates in Sao Paulo, Brazil. Cornea. 2007;26:194-198. 